\title{
Acute Penicillium marneffei infection stimulates host M1/M2a macrophages polarization in BALB/C mice
}

\author{
Xiaoying Dai ${ }^{1}$, Congzheng Mao ${ }^{1}$, Xiuwan Lan², Huan Chen ${ }^{1}$, Meihua Li ${ }^{1}$, Jing Bai ${ }^{1}$, Jingmin Deng ${ }^{1}$, Qiuli Liang ${ }^{1}$, \\ Jianquan Zhang ${ }^{1}$, Xiaoning Zhong ${ }^{1}, Y_{i}$ Liang $^{1}$, Jiangtao Fan ${ }^{3}$, Honglin Luo ${ }^{4^{*}}$ and Zhiyi He ${ }^{1^{*}}$ (D
}

\begin{abstract}
Background: Penicillium marneffei (P. marneffei) is a thermally dimorphic fungus pathogen that causes fatal infection. Alveolar macrophages are innate immune cells that have critical roles in protection against pulmonary fungal pathogens and the macrophage polarization state has the potential to be a deciding factor in disease progression or resolution. The aim of this study was to investigate mouse alveolar macrophage polarization states during P. marneffei infection.

Results: We used enzyme-linked immunosorbent (ELISA) assays, quantitative real-time PCR (qRT-PCR), and Griess, arginase activity to evaluate the phenotypic markers of alveolar macrophages from BALB/C mice infected with $P$. marneffei. We then treated alveolar macrophages from infected mice with $P$. marneffei cytoplasmic yeast antigen (CYA) and investigated alveolar macrophage phenotypic markers in order to identify macrophage polarization in response to $P$. marneffei antigens. Our results showed: i) $P$. marneffei infection significantly enhanced the expression of classically activated macrophage (M1)-phenotypic markers (inducible nitric oxide synthase [iNOS] mRNA, nitric oxide [NO], interleukin-12 [IL-12], tumor necrosis factor-alpha [TNF-a]) and alternatively activated macrophage (M2a)-phenotypic markers (arginase1 [Arg1] mRNA, urea) during the second week post-infection. This significantly decreased during the fourth week post-infection. ii) During P. marneffei infection, CYA stimulation also significantly enhanced the expression of M1 and M2a-phenotypic markers, consistent with the results for $P$. marneffei infection and CYA stimulation preferentially induced M1 subtype.
\end{abstract}

Conclusions: The data from the current study demonstrated that alveolar macrophage M1/M2a subtypes were present in host defense against acute $P$. marneffei infection and that CYA could mimic $P$. marneffei to induce a host immune response with enhanced M1 subtype. This could be useful for investigating the enhancement of host anti-P. marneffei immune responses and to provide novel ideas for prevention of $P$. marneffei-infection.

Keywords: Penicillium marneffei, Immune response, Alveolar macrophages polarization, CYA

\section{Background}

Penicillium marneffei (P. marneffei), discovered in 1956 by Capponi et al., is a thermally dimorphic Penicillium that causes a lethal systemic infection, even though other Penicillium species are usually not pathogenic to humans [1]. Clinically, P. marneffei is one of the most important opportunistic infectious pathogens in Southeast Asia and

\footnotetext{
* Correspondence: 541365548@qq.com; zhiyi-he@163.com

${ }^{4}$ Guangxi Medical University, Nanning, Guangxi 530021, China

'Department of Respiratory Medicine, The First Affiliated Hospital of Guangxi

Medical University, Nanning, Guangxi 530021, China

Full list of author information is available at the end of the article
}

can cause a life-threatening systemic mycosis in immunocompromised individuals [2], and sometimes in immunocompetent individuals [3, 4]. For example, $\mathrm{Hu}$ et al. [5] reviewed 668 cases of $P$. marneffei between 1984 and December 2009 in Mainland China and showed that 99.4\% of cases were reported in the southern part of China (Guangxi and Guangdong provinces), with $87.7 \%$ of these infections occurring in patients with human immunodeficiency virus (HIV). Only $8.5 \%$ of patients did not have HIV. HIV-reduced levels of CD4 $4^{+}$T cells could make host anti-P. marneffei immune responses ineffective, and 
therefore, make $P$. marneffei infection difficult to control in such patients. Therefore, HIV patients successfully treated for P. marneffei infection should receive long-term maintenance therapy to prevent recurrence [6]. Most opportunistic infections, such as Cryptococcus neoformans, Aspergillus fumigatus, and Pneumocystis, rely more heavily on innate immunity when they are T cell deficient. Macrophages are innate immune cells that have critical roles in protection against pulmonary fungal pathogens, including C. neoformans, A. fumigatus, Pneumocystis and Candida albicans [7-10]. Macrophage polarization state has the potential to be a deciding factor in disease progression or resolution [11].

In the environment, $P$. marneffei can grow at $25^{\circ} \mathrm{C}$ as filaments and at $37{ }^{\circ} \mathrm{C}$ as yeast, and cause fatal disseminated and systemic infection in humans or rodent hosts, such as the bamboo rat $[12,13]$. Clinically, P. marneffei infection usually appears in the lung, which could be due to inhalation of airborne P. marneffei conidia [14]. Furthermore, $P$. marneffei conidia are small enough to reach the alveolar spaces [14], leading to activation of alveolar macrophages as the first line of response in the host. A previous study [15] showed that a sublethal $P$. marneffei infection in BALB/c mice triggered a protective $\mathrm{T}$ helper lymphocyte Th1 immune response, as well as interferon-gamma (IFN- $\gamma$ ) expression to activate fungicidal macrophages through the L-arginine-dependent nitric oxide pathway. This indicates that the host immune response against $P$. marneffei infection is mainly mediated by macrophages and T-lymphocytes $[15,16]$. In terms of the immune response, macrophage polarization is phenotypically and functionally plastic in order to respond to cytokine and fungus-sensing environments [17]. Functionally, pro-inflammatory macrophages are termed classically activated macrophages (M1), whereas those that inhibit inflammation and enhance tissue repair are termed alternatively activated macrophages (M2) [18]. M2 macrophages are further subdivided as M2a (after exposure to IL-4 or IL-13), M2b (immune complexes in combination with IL-1 $\beta$ or lipopolysaccharide [LPS]), and M2c (IL-10, transforming growth factor-beta [TGF- $\beta$ ] or glucocorticoids) $[19,20]$. M1 cells can express iNOS to produce NO, and secrete significant amounts of pro-inflammatory cytokines, such as TNF- $\alpha$ and IL-12. M2 macrophages are characterized by expression of the enzyme Arg1, which hydrolyzes Arg1 to ornithine and urea, which are important for cellular proliferation and tissue repair [21]. M2a cells do not express iNOS, but express high levels of Arg1 and low levels of IL-10 [19]. Arg1 and iNOS enzymes can be used to ascertain the pathway of macrophage activation in rodents [22]. Our current study only focused on M1 and M2a macrophages, both of which are associated with antifungal responses [11, 23, 24]. Macrophages can be directed towards the M1 phenotype by Th1-type cytokines such as IFN- $\gamma$, or towards the M2 phenotype by Th2-type cytokines such as IL-4, IL-10, or IL-13 [19, 25]. These $M 1$ and $M 2$ macrophages will promote Th1 and Th2-induced host immune responses, respectively, however, Th1 and Th2 cytokines (e.g., IFN- $\gamma$ or IL-4) can also down-regulate M2 and M1 activity for appropriate immune responses against pathogens [18]. Most research considering macrophage polarization states that result in anti-fungal activity has the potential to provide a novel approach for the treatment of fungal infections. The stimulation of M1 macrophage activation and/or the prevention of M2 macrophage activation have the potential to provide protection against fungal infections, including $C$. neoformans, and A. fumigates [11, 24]. However, enhancing M2a polarization, have a protective role in defense against Pneumocystis infections [23].

P. marneffei cytoplasmic yeast antigen (CYA) is prepared from $P$. marneffei yeast cells and 61-, 54-, and $50-\mathrm{kDa}$ antigens purified from CYA can be used either singly or in combination to detect antibody responses in a large percentage of individuals infected with $P$. marneffei [26]. These antigens have a strong homology ( $87 \%$ identity) with the antioxidant enzyme catalase. Catalase antigens are known to be produced by a number of pathogenic fungi including Histoplasma capsulatum and Aspergillus fumigates and may play a role in inducing immune responses [27, 28]. These studies demonstrated that the CYA could have the ability to activate an immune response.

Alveolar macrophage polarization states during $P$. marneffei infection are unknown. Further, it has not been reported whether CYA crude antigen can stimulate alveolar macrophages. In this study, we utilized a mouse model to investigate alveolar macrophage polarization after P. marneffei infection, as well as the possible role of CYA in alveolar macrophage polarization.

\section{Results \\ Isolation and identification of the GXHCBR P. marneffei strain}

The GXHCBR P. marneffei strain was isolated from lung, liver, and spleen of the bamboo rat. The P. marnef$f e i$ strain was then cultured in potato dextrose agar and grew as a mold at $25{ }^{\circ} \mathrm{C}$. A unique characteristic of $P$. marneffei mold is that it can produce a soluble red pigment that diffuses into the agar (Fig. 1a). A typical mold was observed as hyaline filamentous forms with branches, sometimes with chains of smooth conidia giving the appearance of a brush compatible with $P$. marneffei after Lactophenol cotton blue staining (Fig. 1b). Also these fungi were identified by gold standard DNA sequencing of the fungal internal transcribed spacer (ITS) region (Additional file 1). 


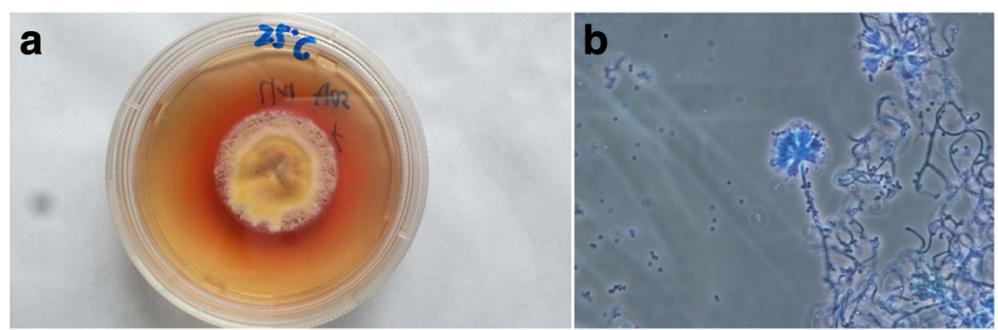

Fig. 1 Identification of P. marneffei strain by culture and Lactophenol cotton blue staining. a, $P$. marneffei strain was cultured in potato dextrose agar: A unique characteristic of $P$. marneffei mold is that it can produce a soluble red pigment that diffuses into the agar at $25^{\circ} \mathrm{C}$. $\mathbf{b}$, Microscopic examination revealed hyaline filamentous forms with branches, sometimes with chains of smooth conidia giving the appearance of a brush compatible with $P$. marneffei by Lactophenol cotton blue staining $(\times 400)$

\section{Confirmation in an animal model and alveolar macrophages}

Lung tissues were resected from $P$. marneffei infected mice and cultured to isolate $P$. marneffei in potato dextrose agar. This formed typical molds in 5-7 days. $P$. marneffei-specific MP1 PCR data confirmed that mold on the plate was the P. marneffei pathogen (Fig. 2a). Alveolar macrophages from infected mice were cultured and stained with Wright's stain, which showed round cells with large and dark nuclei under a light microscope (Fig. 2b). Alveolar macrophage identification was by immunostaining for the macrophage cell marker CD68. CD68+ macrophages were identified by their green fluorescent (Fig. 2c).

\section{Isolation and confirmation of $P$. marneffei CYA}

CYA was extracted from the GXHCBR strain of $P$. mar$n$ effei in the yeast phase. We then confirmed these preparations to be CYA using Western blot. The CYA extracts were subjected to sodium dodecyl sulfate-polyacrylamide gel electrophoresis (SDS-PAGE) and the gels stained with Coomassie brilliant blue with the relative molecular masses of the protein bands observed (Fig. 3a). Proteins were subjected to Western blot analysis using sera from a HIV negative individual with $P$. marneffei infection and the blots identified a 54-kDa antigen (Fig. 3b). Following that, we performed matrix-assisted laser desorption/ ionization time of flight mass spectrometry (MALDI-TOF MS) analysis of these 54-kDa antigens with Mascot software and the national center of biotechnology information (NCBI) non-redundant database from the $P$. marneffei strain (ATCC 18224 / CBS 334.59 / QM 7333). The 54$\mathrm{kDa}$ antigens had a strong homology (81.82\% identity) with the Bifunctional catalase-peroxidase Cat2 (score $>1000$, Table 1).

\section{Differential expression of $\mathrm{M} 1$ and M2a-related cytokines and key enzymes in alveolar macrophages}

First, we prepared the conditioned media from alveolar macrophage cultures and analyzed for cytokines levels using ELISA. The data showed that levels of IL-12 and TNF- $\alpha$ were significantly higher in the conditioned media from alveolar macrophages two weeks postinfection than both the control group and the fourth week post-infection group. Conversely, there was no statistical difference in IL-10 levels among these groups. The above indicators in the fourth week post-infection group were decreased significantly when compare with the second week post-infection group (Fig. 4a). Next, we analyzed key enzymes from alveolar macrophages using

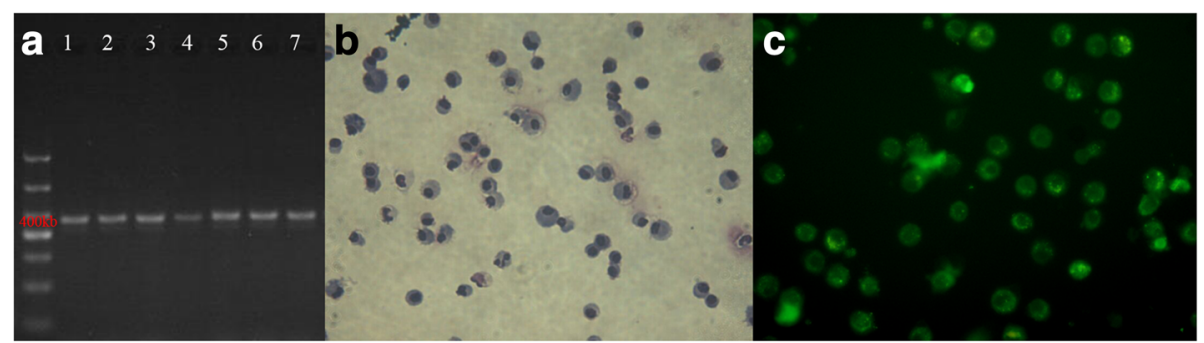

Fig. 2 Confirmation of Animal model and alveolar macrophages. a, The MP1 PCR products were amplified from the mold on the plate of infected mice. Lane 1, PCR products (422 bp) of MP1gene from the GXHCBR P. marneffei strain. Lane 2-4, PCR products (422 bp) of the MP1gene from the molds of two weeks post-infected mice. Lane 5-7, PCR products (422 bp) of the MP1gene from the molds of four weeks post-infected mice. b, Microscopic examination shows round cells with a large and dark nucleus by Wright's staining $(\times 400)$. $\mathbf{c}$, Immunofluorescence for CD68 expression in alveolar macrophages $(\times 400)$ 


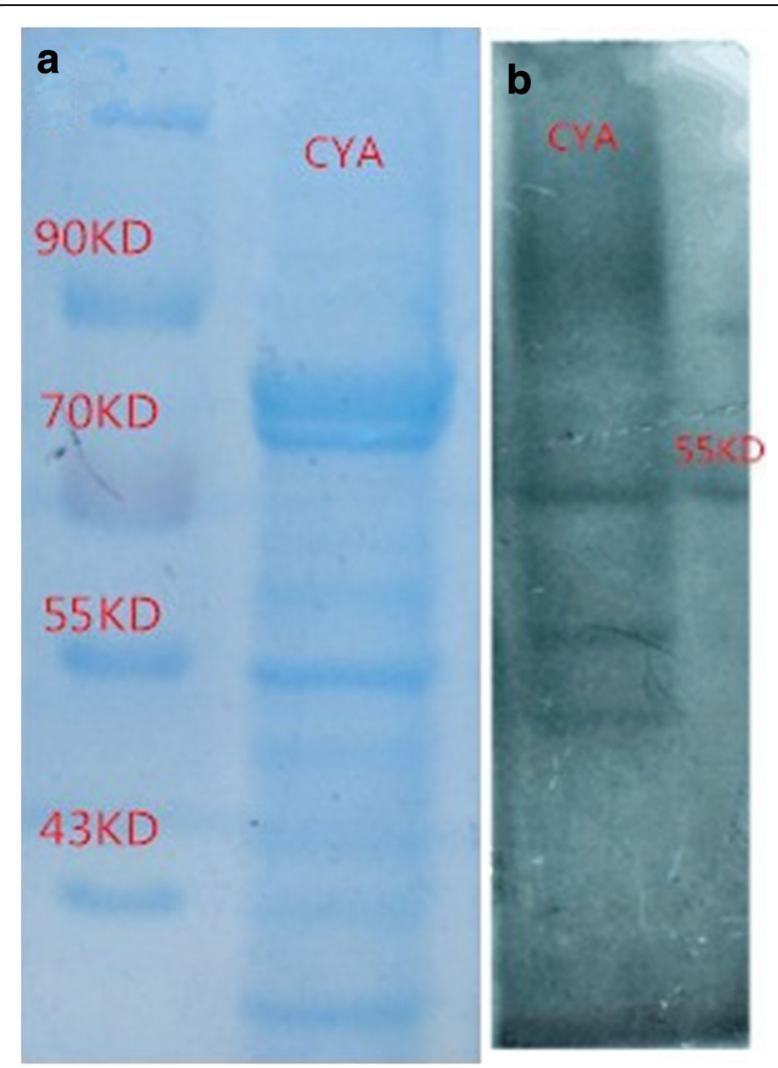

Fig. 3 Identification of CYA by Western blot. a, The SDS-PAGE gel was prepared to separate CYA samples and stained with coomassie brilliant blue. b, CYA samples were assayed by Western blot analysis using sera from a HIV negative individual with $P$. marneffei infection. The most prominent band was identified as the $54-\mathrm{kDa}$ antigen

qRT-PCR, Griess and arginase activity assays. Moreover, similar trends were observed in the relative levels of iNOS and Arg1 mRNA and their activity products NO and urea (Fig. 4 b \& c). These data indicate that alveolar macrophage M1/M2a subtypes were present in host defense against acute $P$. marneffei infection.

\section{Effects of CYA on alveolar macrophage polarization}

To investigate the effects of CYA on alveolar macrophage polarization on $P$. marneffei infected mice, we treated alveolar macrophages with no stimuli as negative control (PM group, PM is an abbreviation of $P$. marneffei), IFN$\gamma+$ LPS as the M1 positive control (PM-IFN- $\gamma+$ LPS group), IL-4 as the M2a positive control (PM- IL-4 group) and CYA (PM-CYA group), respectively. We found that during the second week post-infection, CYA stimulation significantly increased expression of IL-12, iNOS mRNA and $\mathrm{NO}$ when compared to the negative control group and $\mathrm{M} 1$ positive control group, and significantly increased expression of TNF- $\alpha$ when compared to the negative control group. CYA stimulation also significantly increased expression of Arg1 mRNA and urea when compared to the negative control group, however, this was decreased in comparison with the M2a positive group. Similarly, no significant change was found in IL-10 levels among all groups (Fig. 5). During the fourth week post-infection, similar trends were observed whereby CYA stimulation significantly increased expression of M1-related cytokines and key enzymes when compared to the negative control group and M1 positive control group. However, CYA stimulation could not enhance the expression of M2arelated cytokines and key enzymes. In comparison with two weeks post-infection, the above markers significantly decreased in the four weeks post-infection group (Fig. 6). The results suggested that CYA stimulation enhanced M1 and M2a macrophage polarization, which was consistent with the results for $P$. marneffei infection. Also, CYA stimulation preferentially enhanced expression of the M1related cytokines and key enzymes in P. marneffei infected mice.

\section{Discussion}

Systemic mycosis caused by P. marneffei is known to be endemic in South and Southeast Asia. It is often associated with immunocompromised patients, although, in recent years it is increasingly observed in individuals without HIV infection. The different clinical manifestations of $P$. marneffei infection depend on the immune status of the host. The alveolar macrophage is the first line of defense against P. marneffei. The objective of this study was to dissect the polarization states of alveolar macrophages on the regulation of mouse immune responses against $P$. marneffei infection. We found that two weeks of infection with $P$. marneffei significantly induced mouse immune responses including presentation of M1 and M2a macrophages in lung tissues. This decreased significantly in the fourth week of infection. We further confirmed our in vivo data using CYA. During $P$. marneffei infection, the polarization tendency of alveolar macrophages induced by CYA was consistent with the results for $P$. marneffei infection and CYA stimulation preferentially induced the M1 subtype. The data from

Table 1 Proteomic analysis of the 54-kDa antigen

\begin{tabular}{|c|c|c|c|c|c|}
\hline $\begin{array}{l}\text { Antigen } \\
\text { source }\end{array}$ & Accession no. & Protein name and species & Score & Coverage & $\mathrm{MW}[\mathrm{kDa}] / \mathrm{pl}$ \\
\hline GXHCBR & B6QK96 & $\begin{array}{l}\text { Bifunctional catalase-peroxidase Cat2; } \\
\text { Penicillium marneffei (strain ATCC 18224/ } \\
\text { CBS } 334.59 \text { / QM 7333) }\end{array}$ & 1192.13 & 81.82 & $82.3 / 6.95$ \\
\hline
\end{tabular}



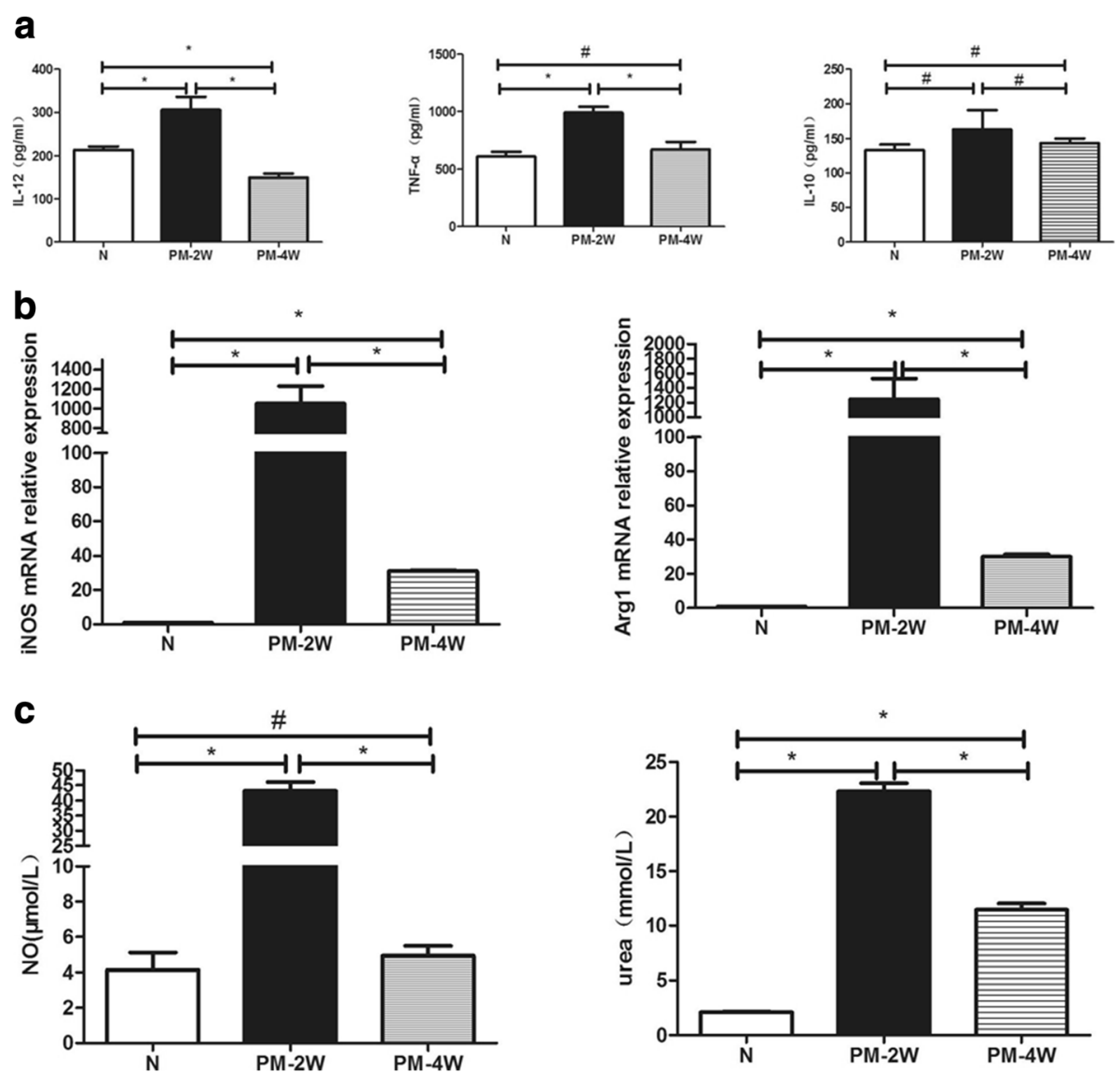

Fig. $4 \mathrm{M} 1$ and M2a-related cytokines and key enzymes expression after P. marneffei infection. a, ELISA. Supernatant from alveolar macrophages of normal control (N) and P. marneffei infected (PM) mice were collected and analyzed for IL-12, TNF-a and IL-10 (M1 and M2a-related cytokines) levels. The data are represented as mean \pm SD $(N=4)$ and analyzed using ANOVA. ${ }^{*} P<0.05$ and ${ }^{\#} P>0.05$. b, qRT-PCR. Alveolar macrophages were collected from normal control (N) and P. marneffei infected (PM) mice and subjected to GRT-PCR for iNOS and Arg1 gene (M1 and M2a-related key enzymes) expression. The data are expressed as mean $\pm \mathrm{SD}(\mathrm{N}=5)$ and analyzed using ANOVA. ${ }^{*} P<0.05$. c, Griess and arginase activity assays. Supernatant from alveolar macrophages of normal control (N) and P. marneffei infected (PM) mice were subjected to NO production using Griess activity assays, and alveolar macrophages were subjected to Urea production using arginase activity assays, respectively. The data are expressed as mean $\pm S D(N=5)$ and analyzed using ANOVA. ${ }^{*} P<0.05$ and ${ }^{\sharp} P>0.05$

the current study demonstrated that M1/M2a macrophages were present in lung tissues in response to acute $P$. marneffei infection, and that CYA could be responsible for $P$. marneffei infection induced host immune responses in mice, enhancing M1 subtype macrophages.

$P$. marneffei infection is endemic in Southeast Asia and China. However, the transmission route of $P$. mar$n$ effei has not yet been verified by large epidemiological investigations. Inhalation of conidia from the environment is thought to be the most likely transmission route. Thus, in the current study, we produced a mouse model of $P$. marneffei infection through intranasal administration. Furthermore, to identify $P$. marneffei infection, we utilized PCR to amplify the MP1 gene, which is a direct indicator for clinical diagnosis of P. marneffei infection according to a previous study [29]. In our current study, all animals were confirmed to be positive for the MP1 gene and $P$. marneffei culture, indicating that they were all successfully infected by $P$. marneffei.

It has been reported that $P$. marneffei is quickly removed by the spleen because the spleen expresses a high level of type 1 cytokines (IL-12 and INF- $\gamma$ ) as well as NO, which plays a crucial role in host defense against $P$. marneffei $[15,30]$. In our current study, the level of M1related iNOS/NO, TNF- $\alpha$, IL-12, as well as M2a-related Arg1/urea were highly induced in alveolar macrophages by acute $P$. marneffei infection. With the progression of infection, their expression was significantly reduced. It has been demonstrated that macrophages can rapidly and uniformly reverse their polarization phenotype in response to different microenvironments and lose or regain their fungicidal function [24]. Moreover, during dimorphic fungus Sporothrix schenckii infection, cell wall peptide-polysaccharide (PPC) of this pathogen was able 

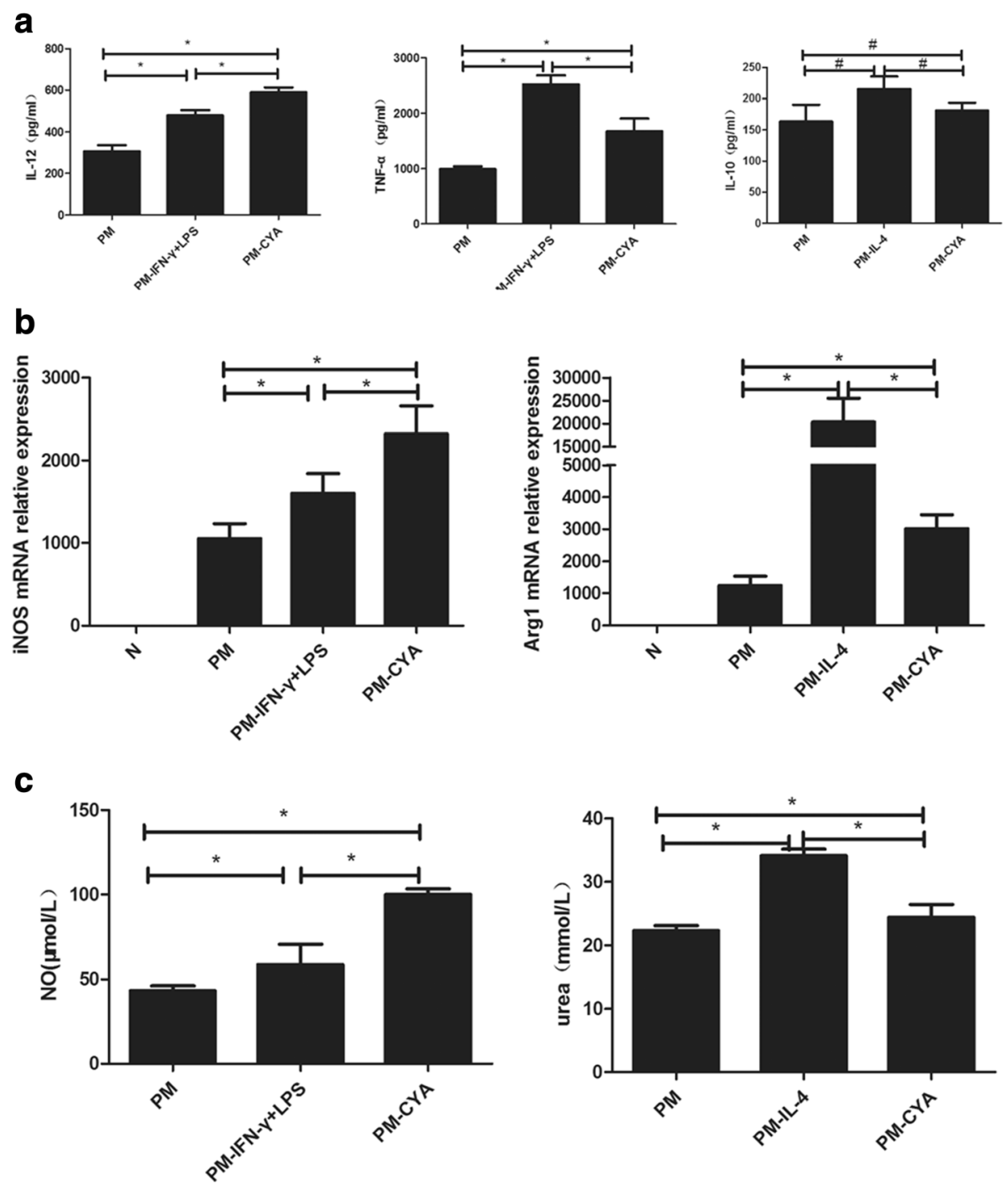

Fig. 5 Effect of CYA on alveolar macrophage polarization in two weeks P. marneffei infected mice. Alveolar macrophages from two weeks $P$. marneffei infected (PM) mice were purified and treated as described in Methods. No stimuli served as a negative control (PM group), IFN- $\gamma+$ LPS were included as the M1 positive control (PM- IFN- - + LPS group), IL-4 was included as the M2a positive control (PM-IL-4 group) and CYA was included as an experimental group (PM-CYA group). a, ELISA. The supernatant from alveolar macrophages with different treatments were collected and analyzed for IL-12, TNF-a and IL-10 (M1 and M2a-related cytokines) levels. The data are represented as Mean \pm SD $(N=4)$ and analyzed using ANOVA. ${ }^{*} P<0.05$ and ${ }^{\#} P>0.05$. b, qRTPCR. Alveolar macrophages after different treatments were collected and subjected to qRT-PCR for iNOS and Arg1 gene (M1 and M2a-related key enzymes) expression. The data are expressed as mean \pm SD $(N=5)$ and analyzed using ANOVA. ${ }^{*} P<0.05$. c, Griess and arginase activity assays. Alveolar macrophages and conditioned media were collected and subjected to Urea and NO production using arginase and Griess activity assays, respectively. The data are expressed as mean \pm SD $(N=5)$ and analyzed using ANOVA. ${ }^{*} P<0.05$ and ${ }^{\#} P>0.05$

to induce IL-12 production, which was consistent with increases in NO production over the same period of time [31]. In our experiment, CYA prepared from $P$. marneffei yeast cells were separated by SDSPAGE and analyzed by Western blot with sera samples from $P$. marneffei-affected individuals, and recognized a series of molecular mass bands. The most important band was at approximately $54-\mathrm{kDa}$. We performed MALDI-TOF MS analysis of these $54-\mathrm{kDa}$ antigens, which had a strong homology $(81.82 \%$ identity $)$ with the Bifunctional catalase-peroxidase Cat2. This was the first investigation into CYA as stimuli on alveolar macrophage polarization. During P. marneffei infection, we found that CYA stimulation could enhance M1-related iNOS mRNA/NO, TNF- $\alpha$, and IL-12 expression, especially in the second week post-infection. CYA stimulation also could enhance M2a-related Arg1 mRNA / urea expression. There was no statistical difference in IL-10 levels among all groups. It was consistent with the results for P. marneffei infection and CYA stimulation had a 

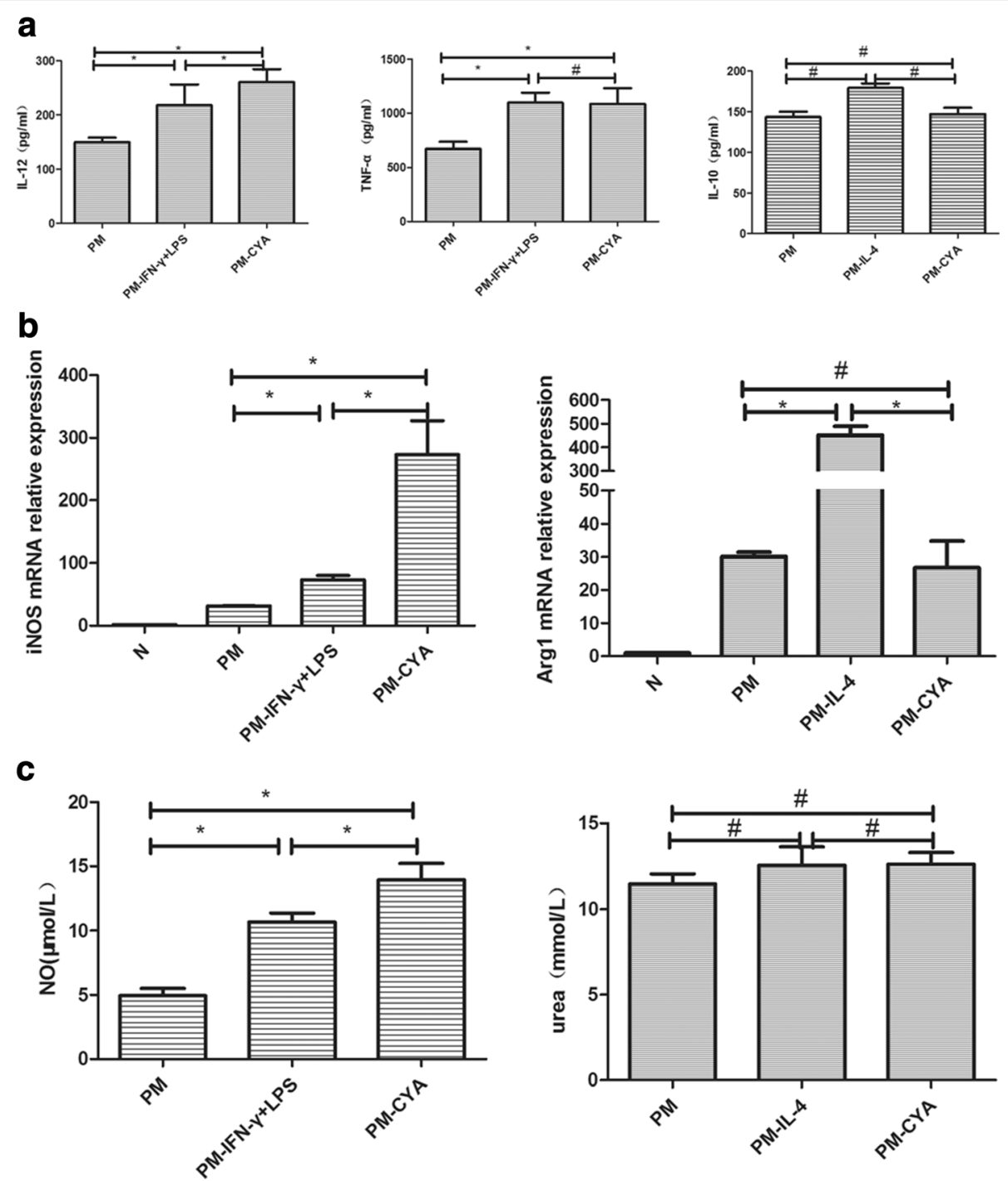

Fig. 6 Effect of CYA on alveolar macrophage polarization in four weeks P. marneffei infected mice. Alveolar macrophages from four weeks P. marneffei infected (PM) mice were purified and treated as described above. $\mathbf{a}$, ELISA. The supernatant from alveolar macrophages with different treatments were collected and analyzed for IL-12, TNF-a and IL-10 (M1 and M2a-related cytokines) levels. The data are represented as Mean \pm SD $(N=4)$ and analyzed using ANOVA. ${ }^{*} P<0.05$ and ${ }^{\#} P>0.05$. b , qRT-PCR. Alveolar macrophages from infected mice were treated with different agents and collected and subjected to qRT-PCR for iNOS and Arg1 gene (M1 and M2a-related key enzymes) expression. The data are expressed as mean \pm SD $(N=5)$ and analyzed using ANOVA. ${ }^{*} P<0.05$ and ${ }^{\#} P>0.05$. c, Griess and arginase activity assays. Alveolar macrophages and conditioned media were collected and subjected to Urea and NO production using arginase and Griess activity assays, respectively. The data are expressed as mean $\pm S D(N=5)$ and analyzed using ANOVA. ${ }^{*} P<0.05$ and ${ }^{\#} P>0.05$

tendency toward increased in M1 subtype macrophages. Our data indicate that CYA could mimic P. marneffei to induce a host immune response and enhance in M1 subtype macrophage polarization, which in turn promotes macrophages to develop enhanced microbicide activity.

Therapeutics targeting modifications of the host response rather than the pathogen would limit selective pressure on the microbe that can lead to drug resistance and increased virulence [11]. Our current study provides proof-of-principle by exploring mouse immune responses against $P$. marneffei infection. Future studies could develop anti-CYA vaccines to prevent $P$. marneffei infection in humans and animals.

\section{Conclusions}

Macrophages play a crucial role in the host anti-P. mar$n e f f e i$ infection response. Our current study demonstrates for the first time to the best of our knowledge that M1/M2a subtype macrophages were present in host defense against acute $P$. marneffei infection, while CYA could mimic $P$. marneffei to induce a host immune response and had a tendency to enhance M1 subtype 
macrophages. Thus, further studies are needed to investigate the enhancement of host anti- $P$. marneffei immune responses and to provide novel ideas for prevention of $P$. marneffei-infection.

\section{Methods}

\section{$P$. marneffei strain and conidia preparation}

The GXHCBR P. marneffei strain was isolated from the organs of the bamboo rat (the lungs, liver, or spleen) from Hechi city, Guangxi Province in Southern China. These fungi were cultured in potato dextrose Agar (PDA) medium (5.0 g potato extract, $20.0 \mathrm{~g}$ dextrose, $20.0 \mathrm{~g}$ agar, and $0.1 \mathrm{~g}$ chloromycetin) at $25{ }^{\circ} \mathrm{C}$ for 7 to 10 days, and identified by morphology and PCR analysis of ITS rDNA sequences. PCR amplification was performed using a Lightcycle 480 system (Roche Applied Science, Basel, Swiss) with primers (ITS1, 5'-TCCGTAGGTGAACCTG CGG-3'; ITS4, 5'-TCCTCCGCTTATTGATATGC-3') at conditions of $95^{\circ} \mathrm{C}$ for $5 \mathrm{~min}$, followed by 30 cycles of $94^{\circ}$ $\mathrm{C}$ for $30 \mathrm{~s}, 52{ }^{\circ} \mathrm{C}$ for $30 \mathrm{~s}$, and $72{ }^{\circ} \mathrm{C}$ for $1 \mathrm{~min}$, and a final extension at $72{ }^{\circ} \mathrm{C}$ for $10 \mathrm{~min}$.

The conidia were collected by flooding the culture surface with phosphate buffered saline (PBS) and counted using a hemocytometer. They were then suspended at a concentration of $5 \times 10^{7}$ colony-forming units $(\mathrm{CFU}) / \mathrm{ml}$.

\section{Preparation and confirmation of $P$. marneffei cytoplasmic yeast antigen (CYA)}

P. marneffei CYA was extracted according to a published study with some modifications [26] . Briefly, the GXHCBR $P$. marneffei strains were cultured in the yeast phase for 7 days at $37{ }^{\circ} \mathrm{C}$ on brain/heart infusion medium (BHIM) broth (10 g tryptone, 5 g sodium chloride, 2.5 g disodium hydrogen phosphate, $2 \mathrm{~g}$ dextrose, and $500 \mathrm{ml}$ beef heart infusion). The medium was then refreshed with BHIM medium and cultured for an additional 7 days. We then harvested the yeast form of the fungus through centrifugation to remove the supernatant. CYA was prepared by mixing packed yeast cells with an equal volume of 0.5mm glass Ballotini beads in PBS. The mixture was then disrupted by sonication (UP100H, Hielscher, Teltow, Germany) and the insoluble debris was removed by centrifugation at $7000 \mathrm{~g}$ for $20 \mathrm{~min}$ at $4{ }^{\circ} \mathrm{C}$. The cytoplasmic antigen solution was then passed through a $0.45 \mu \mathrm{m}$ filter (Corning Inc., Corning, NY, USA). The protein concentration of P. marneffei extracts was then determined by the bicinchoninic acid (BCA) protein assay kit (Cat \#P0018A, Beyotime, Haimen, China), and the extracts were stored at $-80{ }^{\circ} \mathrm{C}$ until use. We then confirmed these preparations to be CYA using Western blot. The extracts were subjected to sodium dodecyl sulfate-polyacrylamide gel electrophoresis (SDS-PAGE) in 10\% SDS-PAGE gels to detect molecular weight, and Western blotting to detect antigens. After SDS-PAGE, the gels were stained with
Coomassie brilliant blue and the relative molecular masses of the protein bands were compared to the prestained protein ladder (Cat \#26617, Thermo Scientific, Rockford, IL, USA). After proteins transferred to the nitrocellulose membrane, the membranes were subjected to Western blot analysis using sera from a HIV negative individual with $P$. marneffei infection at a dilution of 1:250. Following that, goat anti-human immunoglobulin G (IgG) conjugated with peroxidase was added to the membrane at a dilution of 1:2000 and the blots were visualized with enhanced chemiluminescence (Cat \#P0018A, Beyotime) and quantified using the EQ Imaging System (Bio-Rad Laboratories, Inc., Hercules, CA, USA).

\section{MALDI-TOF MS analysis}

54-kDa protein spots of GXHCBR P. marneffei strains were manually excised from SDS-PAGE gels, subjected to in situ digestion with trypsin, and then analyzed using a 4800 Plus (Applied Biosystems, Foster city, CA, USA) according to manufacturer's protocol.

\section{Animals model preparation and confirmation}

A total of 150 five to six-week old male BALB/c mice weighing 20 to $24 \mathrm{~g}$ were purchased from the Laboratory Animal Centre of Guangxi Medical University and were randomly assigned to either the $P$. marneffei uninfected group with 30 mice, two weeks $P$. marneffei-infected group, or four weeks $P$. marneffei-infected group with 60 mice in each group. Mice were infected with the GXHCBR P. marneffei strain according to a published study with some modifications [16]. BALB/c mice were narcotized with diethyl ether and then a sub-lethal suspension of P. marneffei conidia $\left(2.5 \times 10^{6}\right.$ conidia per mouse in $50 \mu \mathrm{l}$ PBS) was slowly dripped into the nose of each mouse and raised for two or four weeks. The negative control mice were without any treatment. The mice received sterilized food and water at the laboratory animal care center. Animal care was in accordance with national and institutional policies for animal health and well-being. The experimental protocol was approved by the Animal Care and Welfare Committee of Guangxi Medical University.

Anesthetized mice were sacrificed at two and four weeks after $P$. marneffei infection and dissected to evaluate $P$. marneffei growth. Part of the lung tissues were removed, plated and cultured in the PDA medium at $25{ }^{\circ} \mathrm{C}$. The molds on the plate were collected for $P$. marneffei specific MP1 PCR amplification with primers (5'-CCACGAA CTCGCCGACATTTC-3' and 5'-CAGAGGAACGACA GGAACGGA-3' to generate a PCR product of $422 \mathrm{bp}$ ) under the conditions of $95{ }^{\circ} \mathrm{C}$ for $5 \mathrm{~min}$ and 30 cycles of $94{ }^{\circ} \mathrm{C}$ for $30 \mathrm{~s}, 55{ }^{\circ} \mathrm{C}$ for $30 \mathrm{~s}$, and $72{ }^{\circ} \mathrm{C}$ for $45 \mathrm{~s}$, followed by a final extension of $72{ }^{\circ} \mathrm{C}$ for $10 \mathrm{~min}$. PCR products were analyzed by $2 \%$ agarose gel electrophoresis and 
images were analyzed by Gel Doc EQ Imaging System (Bio-Rad Laboratories, Inc.).

\section{Alveolar macrophage isolation and culture}

Alveolar macrophages were isolated from bronchoalveolar lavage fluid (BALF) by centrifugation at $300 \mathrm{~g}$ for $15 \mathrm{~min}$ at $4{ }^{\circ} \mathrm{C}$. The cells were then resuspended in roswell park memorial institute 1640 (RPMI-1640) medium (Gibco) supplemented with $100 \mathrm{U} / \mathrm{mL}$ penicillin, $100 \mu \mathrm{g} / \mathrm{mL}$ streptomycin, referred to here as RPMI-1640 complete (RPMI-1640C) medium at a density of $1 \times 10^{6} /$ well and seeded into 12-well plates to allow macrophages to adhere. Macrophages were purified by adherence for $2.5 \mathrm{~h}$ at $37{ }^{\circ} \mathrm{C}$ in a $5 \%$ carbon dioxide (CO2) incubator and yielded $>95 \%$ purity. Then macrophage were stained with Wright stain and immunostaining for CD68 and viewed under an inverted microscope.

The adhered alveolar macrophages were then divided into five groups: $\mathrm{i}$ ) normal group ( $\mathrm{N}$ group) from uninfected mice were cultured in $1 \mathrm{ml}$ of RPMI-1640C medium alone; ii) P. marneffei infected group (PM group) from infected mice were incubated in $1 \mathrm{ml}$ of RPMI1640C medium; iii) M1 positive control group (PM-IFN$\gamma+$ LPS group) from infected mice were incubated in $1 \mathrm{ml}$ of RPMI-1640C medium containing $100 \mathrm{U} / \mathrm{ml} \mathrm{IFN- \gamma \text {and }}$ $5 \mathrm{ng} / \mathrm{ml}$ lipopolysaccharide (LPS); iv) M2a positive control group (PM-IL-4 group) from infected mice were incubated in $1 \mathrm{ml}$ of RPMI-1640C medium containing $100 \mathrm{ng} /$ $\mathrm{ml} \mathrm{IL-4;} \mathrm{and} \mathrm{v)} \mathrm{the} \mathrm{experimental} \mathrm{group} \mathrm{(PM-CYA} \mathrm{group)}$ from infected mice incubated in $1 \mathrm{ml}$ of RPMI-1640C medium containing $50 \mu \mathrm{g} / \mathrm{ml} \mathrm{CYA}$. All of groups were cultured at $37{ }^{\circ} \mathrm{C}$ in $5 \% \mathrm{CO} 2$ for $24 \mathrm{~h}$. At the end of each experiment, the conditioned medium of each group were collected and assayed for nitric oxide and cytokine level, while alveolar macrophages were collected for qRT-PCR and Arg1 activity assay.

\section{ELISA detection of cytokine levels}

Levels of IL-12, TNF- $\alpha$ and IL-10 in alveolar macrophage-conditioned medium were measured using ELISA kits (Cat \#CSB-E07360m, \#CSB-E04594m, and \#CSB-E04741m; CUSABIO, Wuhan, China) according to the manufacturer's instructions.

\section{qRT-PCR detection of iNOS, Arg1mRNA levels}

Total RNA from alveolar macrophages were isolated using the TRIzol reagent (Invitrogen, Carlsbad, CA, USA) to identify iNOS and Arg1 mRNA. RNA samples (1 $\mu \mathrm{g}$ each) were reverse transcribed into cDNA using a reverse transcription kit (Cat \#RR047A; Takara, Dalian, China). The qPCR primers were designed by using Primer Express Software and synthesized by Takara (see Table 2). qPCR was carried out using SYBR Green Master (ROX) reagents (Cat \#RR820A; Takara) in the ABI Stepone Plus (ABI
Table 2 Primer sequences used for qPCR

\begin{tabular}{lll}
\hline Gene & Sequences & Product size (bp) \\
\hline iNOS & 5'-CAAGCACATTTGGGAATGGAGA-3' & 136 \\
& 5'-CAGAACTGAGGGTACATGCTGGAG-3' & \\
Arg1 & 5'-AGCTCTGGGAATCTGCATGG-3' & 125 \\
& 5'-ATGTACACGATGTCTTGGCAGATA-3' & \\
GAPDH & 5'-TGTGTCCGTCGTGGATCTGA-3' & 150 \\
& 5'-TTGCTGTTGAAGTGGAGGAG-3' & \\
T-bet & 5'-CATGGAGAACGGAGAATGGA-3' & 118 \\
& 5'-TGGACAGGGGAAGAGAGCA-3' & \\
GATA-3 & 5'-GGATGTAAGTCGAGGCCCAAG-3' & 117 \\
& 5'-ATTGCAAAGGTAGTGCCCGGTA-3' & \\
B-actin & 5'-CATCCGTAAAGACCTCTATGCCAAC-3' & 171 \\
& 5'-ATGGAGCCACCGATCCACA-3' & \\
\hline
\end{tabular}

Biosystems). The reaction was initiated for $95^{\circ} \mathrm{C}$ for $30 \mathrm{~s}$, followed by 40 cycles of $95{ }^{\circ} \mathrm{C}$ for $5 \mathrm{~s}$ and $60{ }^{\circ} \mathrm{C}$ for $30 \mathrm{~s}$. Data were normalized to GAPDH (reference genes) and calculated using the 2- $\Delta \Delta \mathrm{Ct}$ method.

\section{Griess assay to detect NO level}

$50 \mu \mathrm{l}$ aliquots of alveolar macrophage-conditioned medium were mixed with $50 \mu \mathrm{l}$ of Griess reagent (Applygen Technologies Inc., Beijing, China) and incubated for $10 \mathrm{~min}$ at the room temperature in the dark. The colorimetric reaction was then measured at $540 \mathrm{~nm}$ using a Mutiskan Go microplate reader (Thermo Scientific, Rockford, IL, USA).

\section{Arginase activity assay to detect urea level}

Arginase activity was measured using a method described by Corraliza et al. [32] . Briefly, $1 \times 10^{6}$ alveolar macrophages were lysed with $50 \mu \mathrm{l}$ of $0.1 \%$ Triton X-100 for $30 \mathrm{~min}$ and then added to $50 \mu \mathrm{l}$ of $50 \mathrm{mmol} / \mathrm{L}$ Tris$\mathrm{HCl} / 10 \mathrm{mmol} / \mathrm{L} \mathrm{Cl} 2 \mathrm{Mn} \cdot 4 \mathrm{H} 2 \mathrm{O}(\mathrm{pH} 7.5)$ and incubated at $55{ }^{\circ} \mathrm{C}$ for $10 \mathrm{~min}$. L-arginine hydrolysis was carried out by incubating with $25 \mu \mathrm{l}$ of $0.5 \mathrm{M} \mathrm{L}$-arginine ( $\mathrm{pH}$ 9.7) at $37{ }^{\circ} \mathrm{C}$ for $60 \mathrm{~min}$. The reaction was then stopped with $400 \mu \mathrm{l}$ of stop solution [H2SO4 (96\%)/H3PO4 (85\%)/ $\mathrm{H} 2 \mathrm{O}(1: 3: 7, v / \mathrm{v} / \mathrm{v})]$ and $25 \mu \mathrm{l}$ of $9 \%$ of 2isonitrosopropiophenone. The reactions were incubated at $100{ }^{\circ} \mathrm{C}$ for $45 \mathrm{~min}$ and $100 \mu \mathrm{l}$ of each sample was analyzed using a microplate reader at $540 \mathrm{~nm}$. A standard curve was generated from urea solutions (0-20 mM), which were used to determine the final concentrations.

\section{Statistical analysis}

The data were summarized as the mean \pm standard deviation (SD) of at least three-independent experiments. Statistical analyses were performed using SPSS16.0 (SPSS, Chicago, IL, USA) and the two-sample t-test was carried out to compare difference between groups and one-way analysis of variance (ANOVA) for multiple comparisons. The probability level $\leq 0.05$ was considered as statistically significant. 


\section{Additional files}

Additional file 1: Title of data- Sequences of $P$. marneffei strains using PCR and the sequence of PCR product. Description of data- The GXHCBR $P$. marneffei strains were identified by gold standard DNA sequencing of the fungal ITS region using PCR and the sequence of PCR product. (DOCX $12 \mathrm{~kb})$

Additional file 2: Title of data-raw data. Description of data- The raw data of Fig. 4, Fig. 5 and Fig. 6, please see Tables 1, 2, and 3, respectively. (DOCX 27 kb)

\section{Abbreviations}

ANOVA: One-way analysis of variance; Arg1: Arginase1; BALF: Bronchoalveolar lavage fluid; BCA: Bicinchoninic acid; BHIM: Brain/heart infusion medium; CFU: Colony-forming units; CO2: Carbon dioxide; CYA: Cytoplasmic yeast antigen; ELISA: Enzyme-linked immunosorbent; HIV: Human immunodeficiency virus; IFN- $\gamma$ : Interferon- gamma; IgG: Immunoglobulin G; IL-12: Interleukin-12; iNOS: Inducible nitric oxide synthase; ITS: Internal transcribed spacer; LPS: Lipopolysaccharide; M1: Classically activated macrophage; M2a: Alternatively activated macrophage; MALDI-TOF MS: Matrix-assisted laser desorption/ionization time of flight mass spectrometry; N: Normal; NCBI: National center of biotechnology information; NO: Nitric oxide; PBS: Phosphate buffered saline; PDA: Potato dextrose Agar; PM: P. marneffei; PPC: Peptide-polysaccharide; qRT-PCR: Quantitative real-time PCR; RPMI 1640: Roswell park memorial institute 1640; RPMI-1640C: RPMI1640 complete; SD: Standard deviation; SDS-PAGE: Sodium dodecyl sulfatepolyacrylamide gel electrophoresis; TGF- $\beta$ : Transforming growth factor-beta; TNF-a: Tumor necrosis factor-alpha
\end{abstract}

\section{Acknowledgments}

Not applicable.

\section{Conflict of interest}

The authors declare that they have no conflict of interest.

\section{Funding}

This work was supported in part by grants from Natural Science Foundation of Guangxi Province (\#2016GXNSFAA380270).

\section{Availability of data and materials}

The datasets used and/or analysed during the current study available from the corresponding author on reasonable request. As to the raw data, please refer to the Additional file 2 .

\section{Authors' contributions}

$\mathrm{ZH}, \mathrm{XD}$ and $\mathrm{HL}$ conceived and designed the experiments. $\mathrm{XD}, \mathrm{CM}$ and $\mathrm{HC}$ performed the experiments. $X D$ wrote the manuscript. $X D, C M, Y L, Q L$ analyzed the data. $M L, J B, J D, J Z, X Z$ for critical reading and editing of the manuscript. $X L$ and JF contributed reagents, materials and technical support. All authors reviewed the manuscript. All authors read and approved the final manuscript.

\section{Ethics approval and consent to participate}

The animal work presented in this study was approved by the Animal Care and Welfare Committee of Guangxi Medical University (201504008).

\section{Consent for publication}

Not applicable.

\section{Publisher's Note}

Springer Nature remains neutral with regard to jurisdictional claims in published maps and institutional affiliations.

\section{Author details}

'Department of Respiratory Medicine, The First Affiliated Hospital of Guangxi Medical University, Nanning, Guangxi 530021, China. ${ }^{2}$ Guangxi Colleges and Universities Key Laboratory of Preclinical Medicine Research, Nanning, Guangxi 530021, China. ${ }^{3}$ Department of Gynecology, The First Affiliated Hospital of Guangxi Medical University, Nanning, Guangxi 530021, China. ${ }^{4}$ Guangxi Medical University, Nanning, Guangxi 530021, China.
Received: 23 May 2017 Accepted: 9 August 2017

Published online: 18 August 2017

\section{References}

1. Capponi M, Segretain G, Sureau P. Penicillosis from Rhizomys Sinensis. Bull Soc Pathol Exot Filiales. 1956;49:418-21.

2. Lu S, Hu Y, Lu C, Zhang J, Li X, Xi L. Development of in vitro macrophage system to evaluate phagocytosis and intracellular fate of Penicillium marneffei conidia. Mycopathologia. 2013:176:11-22.

3. Joosten SA, Hannan L, Heroit G, Boerner E, Irving L. Penicillium marneffei presenting as an obstructing endobronchial lesion in an immunocompetent host. Eur Respir J. 2012;39:1540-3

4. Qiu Y, Zhang J, Liu G, Zhong X, Deng J, He Z, Jing B. A case of Penicillium marneffei infection involving the main tracheal structure. BMC Infect Dis. 2014;14:242.

5. Hu Y, Zhang J, Li X, Yang Y, Zhang Y, Ma J, Xi L. Penicillium marneffei infection: an emerging disease in mainland China. Mycopathologia. 2013;175:57-67

6. Supparatpinyo K, Perriens J, Nelson KE, Sirisanthana T. A controlled trial of itraconazole to prevent relapse of Penicillium marneffei infection in patients infected with the human immunodeficiency virus. N Engl J Med. 1998:339:1739-43.

7. Hardison SE, Herrera G, Young ML, Hole CR, Wozniak KL, Wormley FL Jr. Protective immunity against pulmonary cryptococcosis is associated with STAT1-mediated classical macrophage activation. J Immunol. 2012;189:4060-8.

8. Bhatia S, Fei M, Yarlagadda M, Qi Z, Akira S, Saijo S, Iwakura Y, van Rooijen N, Gibson GA, St Croix CM, et al. Rapid host defense against Aspergillus fumigatus involves alveolar macrophages with a predominance of alternatively activated phenotype. PLoS One. 2011;6:e15943.

9. Limper AH, Hoyte JS, Standing JE. The role of alveolar macrophages in pneumocystis carinii degradation and clearance from the lung. J Clin Invest. 1997;99:2110-7

10. Reales-Calderon JA, Aguilera-Montilla N, Corbi AL, Molero G, Gil C. Proteomic characterization of human proinflammatory M1 and antiinflammatory M2 macrophages and their response to Candida Albicans. Proteomics. 2014;14:1503-18.

11. Leopold Wager CM, Wormley FL Jr. Classical versus alternative macrophage activation: the Ying and the Yang in host defense against pulmonary fungal infections. Mucosal Immunol. 2014;7:1023-35.

12. Pasricha S, Payne M, Canovas D, Pase L, Ngaosuwankul N, Beard S, Oshlack A, Smyth GK, Chaiyaroj SC, Boyce KJ, Andrianopoulos A. Cell-type-specific transcriptional profiles of the dimorphic pathogen Penicillium marneffei reflect distinct reproductive, morphological, and environmental demands. G3 (Bethesda). 2013:3:1997-2014.

13. Nierman WC, Fedorova-Abrams ND, Andrianopoulos A. Genome sequence of the AIDS-associated pathogen Penicillium marneffei (ATCC18224) and its near taxonomic relative Talaromyces Stipitatus (ATCC10500). Genome Announc. 2015:3

14. Lau SK, Tse H, Chan JS, Zhou AC, Curreem SO, Lau CC, Yuen KY, Woo PC. Proteome profiling of the dimorphic fungus Penicillium marneffei extracellular proteins and identification of glyceraldehyde-3-phosphate dehydrogenase as an important adhesion factor for conidial attachment. FEBS J. 2013:280:6613-26.

15. Sisto F, Miluzio A, Leopardi O, Mirra M, Boelaert JR, Taramelli D. Differential cytokine pattern in the spleens and livers of BALB/C mice infected with Penicillium marneffei: protective role of gamma interferon. Infect Immun. 2003;71:465-73.

16. Kudeken N, Kawakami K, Kusano N, Saito A. Cell-mediated immunity in host resistance against infection caused by Penicillium marneffei. J Med Vet Mycol. 1996:34:371-8.

17. de Souza SC, Tavares AH, Sousa Jeronimo M, Soares de Lima Y, da Silveira Derengowski L, Bocca AL, Silva-Pereira I. The Effects of Paracoccidioides brasiliensis Infection on GM-CSF- and M-CSF-Induced Mouse Bone MarrowDerived Macrophage from Resistant and Susceptible Mice Strains. Mediators Inflamm. 2015;2015:605450.

18. Mills CD. M1 and M2 macrophages: oracles of health and disease. Crit Rev Immunol. 2012;32:463-88.

19. Martinez FO, Sica A, Mantovani A, Locati M. Macrophage activation and polarization. Front Biosci. 2008;13:453-61.

20. Cassetta L, Cassol E, Poli G. Macrophage polarization in health and disease ScientificWorldJournal. 2011;11:2391-402. 
21. Liu YC, Zou XB, Chai YF, Yao YM. Macrophage polarization in inflammatory diseases. Int J Biol Sci. 2014;10:520-9.

22. Thomas AC, Mattila JT. "of mice and men": arginine metabolism in macrophages. Front Immunol. 2014;5:479.

23. Nelson MP, Christmann BS, Werner JL, Metz AE, Trevor JL, Lowell CA, Steele C. $\mathrm{IL}-33$ and $\mathrm{M} 2 \mathrm{a}$ alveolar macrophages promote lung defense against the atypical fungal pathogen pneumocystis murina. J Immunol. 2011;186:2372-81.

24. Davis MJ, Tsang TM, Qiu Y, Dayrit JK, Freij JB, Huffnagle GB, Olszewski MA. Macrophage M1/M2 polarization dynamically adapts to changes in cytokine microenvironments in Cryptococcus Neoformans infection. MBio. 2013;4:e00264-13.

25. Mosser DM. The many faces of macrophage activation. J Leukoc Biol. 2003;73:209-12.

26. Jeavons L, Hamilton AJ, Vanittanakom N, Ungpakorn R, Evans EG, Sirisanthana T, Hay RJ. Identification and purification of specific Penicillium marneffei antigens and their recognition by human immune sera. J Clin Microbiol. 1998:36:949-54.

27. Hamilton AJ, Bartholomew MA, Figueroa J, Fenelon LE, Hay RJ. Evidence that the $\mathrm{M}$ antigen of Histoplasma capsulatum var. capsulatum is a catalase which exhibits cross-reactivity with other dimorphic fungi. J Med Vet Mycol. 1990;28:479-85.

28. Lopez-Medrano R, Ovejero MC, Calera JA, Puente P, Leal F. An immunodominant 90-kilodalton Aspergillus fumigatus antigen is the subunit of a catalase. Infect Immun. 1995;63:4774-80.

29. Cao L, Chan CM, Lee C, Wong SS, Yuen KY. MP1 encodes an abundant and highly antigenic cell wall mannoprotein in the pathogenic fungus Penicillium marneffei. Infect Immun. 1998;66:966-73.

30. Kudeken N, Kawakami K, Saito A. Different susceptibilities of yeasts and conidia of Penicillium marneffei to nitric oxide (NO)-mediated fungicidal activity of murine macrophages. Clin Exp Immunol. 1998;112:287-93.

31. Alegranci P, de Abreu Ribeiro LC, Ferreira LS, Negrini Tde C, Maia DC, Tansini A, Goncalves AC, Placeres MC, Carlos IZ. The predominance of alternatively activated macrophages following challenge with cell wall peptide-polysaccharide after prior infection with Sporothrix Schenckii. Mycopathologia. 2013;176:57-65.

32. Corraliza IM, Campo ML, Soler G, Modolell M. Determination of arginase activity in macrophages: a micromethod. J Immunol Methods. 1994;174:231-5

\section{Submit your next manuscript to BioMed Central and we will help you at every step:}

- We accept pre-submission inquiries

- Our selector tool helps you to find the most relevant journal

- We provide round the clock customer support

- Convenient online submission

- Thorough peer review

- Inclusion in PubMed and all major indexing services

- Maximum visibility for your research

Submit your manuscript at www.biomedcentral.com/submit 